\title{
Ứng dụng mô hình TRAM để nghiên cứu các yếu tố ảnh hưởng đến ý định sử dụng mobile video trong học tập trụ̣c tuyến
}

\section{Applying TRAM Model in studying factors influencing the intention to use mobile video for online learning}

\author{
Huỳnh Thị Minh Châu ${ }^{1 *}$ \\ ${ }^{1}$ Trường Đại học Bách Khoa, Đại học Quốc Gia Thành phố Hồ Chí Minh, Việt Nam \\ *Tác giả liên hệ, Email: htmchau@hcmut.edu.vn
}

\begin{tabular}{l} 
THÔNG TIN \\
\hline DOI:10.46223/HCMCOUJS. \\
econ.vi.14.1.497.2019 \\
Ngày nhận: 24/11/2018 \\
Ngày nhận lại: 19/12/2018 \\
Duyệt đăng: 14/01/2019
\end{tabular}

\section{Tù khóa:}

học tập trực tuyến, mô hình TRAM, mobile video, ý định sử dụng công nghệ

\section{TÓM TÁT}

Học tập trực tuyến bằng mobile video đã trở nên ngày càng phổ biến trong lĩnh vực giáo dục. Hiểu được ý định sử dụng mobile video trong học tập trực tuyến giúp các nhà kinh doanh dự đoán tiềm năng tiêu thụ các sản phẩm của thị trường này. Dựa trên mô hình TRAM, bài báo này đề xuất một mô hình nghiên cứu các yếu tố ảnh hưởng lên ý định sử dụng mobile video để học tập trực tuyến và tiến hành một khảo sát định lượng trên sinh viên Đại học Bách khoa Thành phố Hồ Chí Minh. Kết quả thu được: (1) một bộ thang đo của 4 khái niệm "sự sẵn sàng công nghệ", "cảm nhận về tính dễ sử dụng", "cảm nhận về tính hữu ích", "ý định sử dụng công nghệ" gồm 18 biến quan sát đạt độ tin cậy và độ giá trị; (2) một mô hình cấu trúc tuyến tính thể hiện 5 mối quan hệ quan trọng: sự sẵn sàng công nghệ có tác động tích cực lên cảm nhận về tính dễ sử dụng và cảm nhận về tính hữu ích; cảm nhận về tính dễ sử dụng có tác động tích cực lên cảm nhận về tính hữu ích; và ý định sử dụng mobile video để học tập trực tuyến bị ảnh hưởng tích cực bởi cảm nhận về tính dễ sử dụng và cảm nhận về tính hữu ích.

ABSTRACT
Online learning with mobile videos has become increasingly
popular in the field of education. Understanding the intention to
use mobile videos in online learning can help businesses anticipate
the potential of consuming products for this market. Basing on the
TRAM model, this research proposes a model of factors
influencing the intention to use mobile videos for online learning,
then conducts a quantitative survey on students of Ho Chi Minh
City University of Technology. The results are: (1) an 18-item
scale of 4 concepts "technology readiness", "perceived ease of
use", "perceived usefulness", "intention to use technology" which
achieve reliability and validity; and (2) a structural model which
represents 5 important relationships: technology readiness that has
positive impacts on both the perceived ease of use and the


Keywords:

intention to use technology, mobile video, online learning, TRAM model perceived usefulness; the perceived ease of use which has a positive effect on the perceived usefulness; and the intention to use mobile videos for online learning which is strongly influenced by both the perceived ease of use and the perceived usefulness.

\section{Giới thiệu}

Internet xuất hiện tại Việt Nam từ năm 1997, và đến nay số người dùng đang gia tăng nhanh chóng. Sự đa dạng và phong phú của dịch vụ internet tại Việt Nam góp phần tạo lập cộng đồng, thúc đẩy hoạt động của các tổ chức xã hội, nâng cao năng lực sản xuất và thương mại, và cải thiện đời sống người dân. Bên cạnh đó, Internet cũng tạo nên môi trường thuận lợi cho việc tiếp cận thông tin trong xã hội, sản xuất kinh doanh, truyền thông, giải trí, và đặc biệt là học tập. Thực tế cho thấy, Internet ngày càng chi phối các hoạt động giáo dục. Theo University World News, châu Á là thị trường lớn thứ hai của giáo dục trực tuyến, được dự báo sẽ đạt 12,1 tỷ USD vào năm 2018 (Báo điện tử Tri Thức Trẻ, 2017). Trong vòng 5 năm (2011-2016), Việt Nam là quốc gia đứng đầu trong số 7 quốc gia châu Á có tên trong top 10 thị trường giáo dục trực tuyến phát triển nhanh nhất, vượt cả Thái Lan và Trung Quốc (AUM Việt Nam, 2017).

Sự phát triển đa dạng của điện thoại thông minh làm cho việc áp dụng thiết bị này vào hoạt động giáo dục càng được quan tâm. Học tập trực tuyến qua điện thoại thông minh được hiểu là sử dụng điện thoại thông minh để truy cập vào các nội dung học tập và các nguồn thông tin (Haag, 2011). Cùng với sự bùng phát số lượng người dùng điện thoại thông minh như hiện nay, nhất là trong giới trẻ (Iqbal \& Bhatti, 2015; Rivera \& van der Meulen, 2014), số lượng người tham gia học trực tuyến thông qua điện thoại thông minh cũng ngày càng tăng theo. Hầu như lúc nào điện thoại thông minh cũng ở bên cạnh người học, nên việc học trên điện thoại thông minh có thể diễn ra mọi lúc mọi nơi miễn kết nối với Internet. Nhờ điện thoại thông minh, việc học tập trực tuyến trở nên tiện lợi hơn vì giúp giảm thiểu việc quản lý thời gian, dễ tiếp cận các khóa đào tạo bắt buộc và có thể giúp nâng cao sự sẵn sàng của người học.

Từ năm 2000, chính phủ Việt Nam đã xác định rằng học tập trực tuyến có tiềm năng thúc đẩy sự tăng trưởng của nền giáo dục. Xây dựng môi trường học tập trực tuyến được quan tâm chú ý và đưa vào triển khai trong nhiều trường đại học ở Việt Nam với phạm vi, mức độ khác nhau (Báo Nhân Dân, 2017). Ambient Insight (2014) dự báo rằng, Việt Nam sẽ trở thành một trong 10 quốc gia có tỷ lệ tăng trưởng học tập trực tuyến qua điện thoại thông minh hàng đầu thế giới cũng như ở khu vực châu Á.

Trong số các phương pháp thiết kế chương trình học tập trực tuyến qua điện thoại thông minh, mobile video là một trong những xu thế hiện nay. Các khóa học trực tuyến bằng mobile video trên điện thoại thông minh được hiểu là các khóa học sử dụng tài liệu giảng dạy dạng video được đăng tải trên web và người học có thể tiếp cận bằng các thiết bị có kết nối internet, trong đó có điện thoại thông minh. Hầu hết các lý thuyết về giáo dục cho rằng video mang lại hiệu quả cao hơn so với các phương tiện khác (Carter, 1996; Hastings \& Tracey, 2005; Van Der Molen \& Van Der Voort, 2000). Tại Việt Nam, học tập trực tuyến bằng mobile video trên điện thoại thông minh đang là một trong những hình thức được ưa chuộng (Báo Dân Trí, 2017). 
Nhiều nhà nghiên cứu đã xem xét sự sẵn lòng của sinh viên đối với học tập trực tuyến qua điện thoại thông minh (Cheon, Lee, Crooks, \& Song, 2012; Hussin, Manap, Amir, \& Krish, 2012; Iqbal \& Bhatti, 2015; Mahat, Ayub, \& Luan, 2012) nhưng khái niệm về sự sẵn lòng này vẫn còn tiếp tục phát triển (Khaddage \& Knezek, 2013; Khaddage et al., 2015), và chưa có nhiều nghiên cứu về ý định của người học trong việc sử dụng mobile video cho học tập trực tuyến. Do đó, bài báo này được tiến hành nhằm tìm hiểu một số tiền tố của ý định sử dụng mobile video để học tập trực tuyến, với mong muốn cung cấp một tài liệu tham khảo hữu ích cho các nhà quản lý và nhà nghiên cứu. Quá trình nghiên cứu gồm 2 bước: (1) tổng hợp các tài liệu có trước để đề xuất một mô hình nghiên cứu dựa trên mô hình TRAM về các yếu tố ảnh hưởng đến ý định sử dụng mobile video để học tập trực tuyến; (2) tiến hành khảo sát định lượng trên đối tượng là sinh viên Đại học Bách khoa TP.HCM để thu thập dữ liệu dùng cho các phân tích EFA, CFA và SEM.

\section{Cơ sở lý thuyết}

\subsection{Mô hìn TRAM}

Mô hình sẵn sàng chấp nhận công nghệ (TRAM) được đề xuất bởi Lin, Shih, và Sher (2007), là kết quả của việc kết hợp mô hình chấp nhận công nghệ (TAM) của Davis (1989) với khái niệm sự sẵn sàng công nghệ (TR) của Parasuraman (2000). Trước hết, trong TAM, ý định sử dụng công nghệ (IU) là khả năng một người sẽ áp dụng một công nghệ nào đó, sẽ dẫn đến hành vi áp dụng hay sử dụng một công nghệ nhất định (Davis, 1989). TAM cho rằng sự chấp nhận hệ thống mới được xác định bởi người dùng có ý định sử dụng hệ thống, ý định này bị ảnh hưởng bởi sự tin tưởng của người dùng về tính dễ sử dụng và tính hữu ích của hệ thống. Nhiều nhà nghiên cứu đã sử dụng TAM làm cơ sở để nghiên cứu việc học tập trực tuyến qua điện thoại thông minh của sinh viên đại học (Almaiah \& Alismaiel, 2018; Huang, SánchezPrieto, Teo, Olmos-Migueláñez, \& García-Peñalvo, 2018; Sánchez-Prieto, Olmos-Migueláñez, \& García-Peñalvo, 2017a; Sánchez-Prieto, Olmos-Migueláñez, \& García-Peñalvo, 2017b; Scherer, Siddiq, \& Tondeur, 2019).

Trong khi đó, $\mathrm{TR}$ đại diện cho xu hướng mọi người nắm lấy và sử dụng công nghệ mới để hoàn thành mục tiêu trong cuộc sống gia đình và tại nơi làm việc (Parasuraman, 2000). Nó là tổng thể trạng thái tâm trí của một người có khuynh hướng thiên về sử dụng công nghệ mới. Chỉ số sẵn sàng công nghệ (TRI) được sử dụng để đo lường TR dựa trên bốn đặc điểm: lạc quan, đổi mới, khó chịu và bất an (Parasuraman, 2000). Trong đó, sự lạc quan đề cập đến niềm tin là công nghệ giúp gia tăng việc kiểm soát, tính linh hoạt, khả năng học tập và sự hiệu quả. Sự đổi mới chỉ ra xu hướng tiên phong về công nghệ và lãnh đạo về tư tưởng. Sự khó chịu nói đến sự thiếu kiểm soát đối với công nghệ. Cuối cùng, sự bất an cho thấy sự nghi ngờ về công nghệ và sự hoài nghi về khả năng hoạt động đúng đắn của nó. Bốn đặc điểm này thường khác nhau giữa các cá nhân và giữa các kiểu công nghệ khác nhau.

Kết hợp TAM và TR, TRAM giải thích tại sao những người có TR cao không phải lúc nào cũng chấp nhận sử dụng các tiện ích công nghệ cao có sẵn trên thị trường, bởi vì cảm nhận về tính dễ sử dụng $(\mathrm{PE})$ và cảm nhận về tính hữu ích $(\mathrm{PU})$ cũng ảnh hưởng đến quá trình ra quyết định chấp nhận sự đổi mới. $\mathrm{PE}$ là mức độ mà một người tin rằng không cần bỏ ra nhiều 
nỗ lực để sử dụng một công nghệ (Davis, 1989). PU là mức độ mà một người tin rằng sử dụng một hệ thống cụ thể sẽ nâng cao hiệu quả công việc của mình (Davis, 1989). Nhiều nghiên cứu đã sử dụng TRAM để tập trung vào phân tích mối quan hệ giữa các đặc điểm cá nhân, sự chấp nhận công nghệ và sự sẵn sàng của người dùng để sử dụng một công nghệ mới (Adiyarta, Napitupulu, Nurdianto, Rahim, \& Ahmar, 2018; Chung, Lee, \& Choi, 2015; Iqbal \& Bhatti, 2015; Jin, 2013; Larasati \& Santosa, 2017; Lundberg, 2017).

\subsection{Mô hình nghiên cúu đề xuất}

Trong bài báo này, TRAM được áp dụng để nghiên cứu một số yếu tố ảnh hưởng lên ý định sử dụng mobile video cho học tập trực tuyến (xem Hình 1). Nhiều tài liệu có trước đã cho thấy tác động tích cực của $P E$ và PU lên IU (Gruzd, Staves, \& Wilk, 2012; Guo \& Barnes, 2012; Hong, Thong, Chasalow, \& Dhillon, 2011; Lian, Yen, \& Wang, 2014; Pynoo et al., 2011; Venkatesh, Thong, Chan, Hu, \& Brown, 2011). Vì vậy, tác giả có căn cứ để suy luận rằng nếu người học càng cảm nhận rằng sử dụng mobile video là dễ dàng và sử dụng mobile video sẽ làm kết quả học tập tốt hơn, thì họ càng có ý định sử dụng mobile video để học tập trực tuyến. Nói cách khác, các giả thuyết sau sẽ được kiểm tra: (H1) Cảm nhận về tính dễ sủ dụng của mobile video có tác động tích cực lên ý định sử dụng mobile video để học tập trục tuyến; và (H2) Cảm nhận về tính hữu ích của mobile video có tác động tích cục lên ý định sư dụng mobile video để học tập trực tuyến.

Bên cạnh đó, TRAM đề xuất một mối tương quan tích cực đáng kể giữa $\mathrm{PE}$ và $\mathrm{PU}$, nghĩa là nếu bất kỳ người nào thấy một công nghệ dễ sử dụng hơn, họ sẽ có thái độ tích cực hơn đối với tính hữu ích của nó. Các nghiên cứu về việc áp dụng công nghệ mới như ngân hàng trực tuyến, thương mại điện tử, các hệ thống e-learning, internet vạn vật... đã chứng minh tác động tích cực của PE lên PU (Baturay, Gökçearslan, \& Ke, 2017; Das, Dash, Sahoo, \& Mohanty, 2017; Liew et al., 2017; Park, Cho, Han, \& Kwon, 2017; Wingo, Ivankova, \& Moss, 2017), do đó, giả thuyết sau sẽ được kiểm tra: (H3) Cảm nhận về tính dễ sủ dụng của mobile video có tác động tích cực lên cảm nhận về tính hưu ích của mobile video.

Trong khi đó, TR là một dự đoán mạnh mẽ cho các ý định hành vi liên quan đến công nghệ (Parasuraman, 2000; Parasuraman \& Colby, 2015). Hầu hết các nghiên cứu về TR đều cho thấy các cá nhân có $T R$ cao thì có xu hướng chấp nhận và sử dụng công nghệ cao (Crosbie, Broderick, Short, Charlesworth, \& Dawood, 2018; Larasati \& Santosa, 2017; Parasuraman, 2000; Parasuraman \& Colby, 2015). TR được chứng minh là có ảnh hưởng tích cực lên ý định khám phá công nghệ (Maruping, Magni, Caporarello, \& Basaglia, 2008; Maruping \& Magni, 2012). Những người học mà có mức độ tự tin về máy vi tính cao hơn thì động lực sử dụng máy vi tính để học tập trực tuyến sẽ cao hơn so với những người khác (Iqbal \& Bhatti, 2015). Do đó giả thuyết sau sẽ được kiểm tra: (H4) Sư sã̃n sàng công nghệ có tác động tích cực lên ý định sử dụng mobile video để học tập trục tuyến.

Ngoài ra, những người học khác nhau có phong cách học tập khác nhau, cũng như sở thích của họ đối với phương pháp học là khác nhau. Một số người học thoải mái hơn trong môi trường trực tuyến so với những người khác, trong khi một số người thích sử dụng công nghệ

mới hơn nếu họ có khả năng và tự tin trong việc hoàn thành nhiệm vụ bằng cách sử dụng công 
nghệ đó. Trong nghiên cứu của Christensen và Knezek (2017), những người lạc quan thì đánh giá điện thoại di động dễ sử dụng cho mục tiêu học tập, còn trong nghiên cứu của Irby (2017), những học sinh trung học nào có niềm tin tươi sáng về công nghệ và là những người thích sự đổi mới thì cho rằng công nghệ hỗ trợ học tập là hữu ích. Trong nghiên cứu của Crosbie và cộng sự (2018), những cư dân ít lo lắng và thích công nghệ thì đánh giá cao sự hữu ích của các hệ thống quản lý năng lượng trong các tòa nhà, còn trong nghiên cứu của Howard, Chan, và Caputi (2015), những giáo viên có đủ niềm tin lạc quan về máy vi tính xách tay sẽ cảm nhận việc giảng dạy bằng máy vi tính xách tay là dễ dàng và mang lại nhiều lợi ích. Do đó, các giả thuyết sau sẽ được kiểm tra: (H5) Sự sã̃n sàng công nghệ có tác động tích cực lên cảm nhận về tính dễ sử dụng của mobile video; và (H6) Sụ sẵn sàng công nghệ có tác động tích cưc lên tính hũu ích của mobile video.

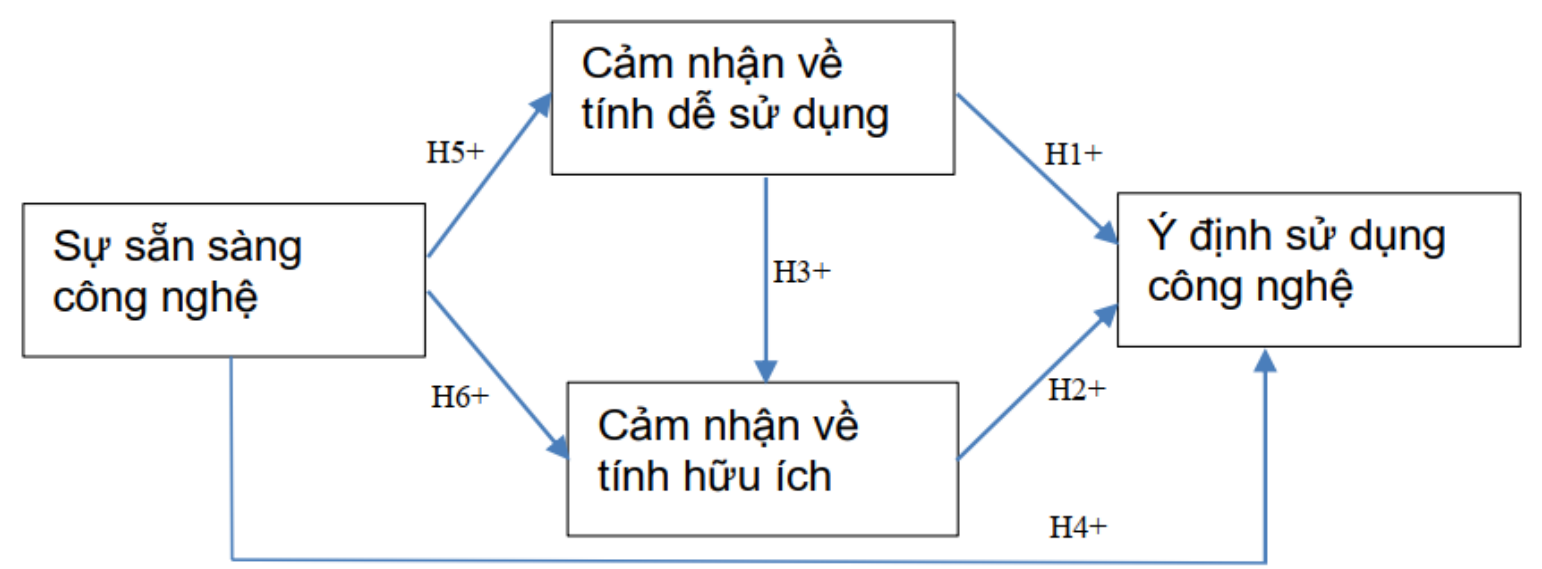

Hình 1. Mô hình nghiên cứu đề xuất về các yếu tố ảnh hưởng đến ý định sử dụng mobile video để học tập trực tuyến

\section{Phương pháp nghiên cứu}

Một khảo sát định lượng được thực hiện với Bảng câu hỏi gồm 3 câu hỏi về nhân khẩu học (Giới tính, Loại chương trình được đào tạo; Loại ngôn ngữ của chương trình đào tạo) và 30 câu hỏi đo lường. Trong đó, 6 câu hỏi để đo cảm nhận về tính dễ sử dụng của mobile video (dựa trên Davis, 1989) mã hóa từ PE1 - > PE6, 6 câu hỏi để đo cảm nhận về tính hữu ích của mobile video (dựa trên Davis, 1989) mã hóa từ PU1 -> PU6, 2 câu hỏi để đo ý định sử dụng mobile video trong học tập trực tuyến (dựa trên Lin et al., 2007) mã hóa từ IU1 -> IU2, 16 câu hỏi để đo sự sẵn sàng công nghệ (dựa trên Parasuraman \& Colby, 2015) mã hóa từ TR1->TR16. Đáp viên được yêu cầu cho biết mức độ từ rất không đồng ý (1) đến rất đồng ý (5) (thang đo Likert 5 điểm). Lấy mẫu thuận tiện bằng cách phát Bảng câu hỏi cho sinh viên Trường Đại học Bách khoa TP.HCM ngay tại trường học hoặc chia sẻ link trên internet. Số bản hợp lệ thu về là 182, lớn hơn 5 lần số biến đo lường (đạt yêu cầu về cỡ mẫu tối thiểu của Hair, Tatham, \& Black, 1998). Dữ liệu được phân tích bằng phần mềm SPSS 23.0 và AMOS 23.0. 


\section{Kết quả nghiên cứu}

\subsection{Thống kê mô tả}

Trong mẫu, có 67 sinh viên nữ (chiếm 37\%), 115 sinh viên nam (chiếm 63\%); có 138 sinh viên được đào tạo theo chương trình $\mathrm{Kỹ} \mathrm{sư} \mathrm{(chiếm} \mathrm{76 \% ),} 44$ sinh viên được đào tạo theo chương trình Cử nhân (chiếm 24\%); có 35 sinh viên được đào tạo bằng tiếng Anh (chiếm 19\%) và 147 sinh viên được đào tạo bằng tiếng Việt (chiếm $81 \%$ ).

\subsection{Mô hình đo lưòng}

\subsubsection{Phân tích nhân tố khám phá}

Kiểm tra độ tin cậy bằng hệ số Cronbach's Alpha, kết quả cho thấy mỗi thang đo đều lớn hơn 0,6 , trong mỗi thang đo, các biến đều có hệ số tương quan biến tổng $>0,3$, vì vậy không có biến nào bị loại khỏi thang đo (Cortina, 1993; Nunnally, 1978). Tiến hành phân tích nhân tố khám phá (EFA) trên 30 biến đo lường, sử dụng phương pháp trích nhân tố PAF với phép quay không vuông góc Promax, hệ số lần chạy thứ nhất cho hệ số $\mathrm{KMO}=0,718$ nằm trong giới hạn $[0,5 ; 1](\mathrm{p}=0,000)$, vì vậy, tập dữ liệu thích hợp để phân tích EFA. Biến (TR1) tải lên 2 nhân tố với hệ số tải lần lượt là 0,606 và 0,521 , biến (TR7) tải lên 2 nhân tố với hệ số tải lần lượt là 0,562 và 0,475 , nên loại 2 biến này khỏi thang đo, tập dữ liệu còn lại 28 biến. Phân tích EFA lần thứ hai cho hệ số $\mathrm{KMO}=0,737$ nằm trong giới hạn $[0,5 ; 1](\mathrm{p}=0,000)$, vì vậy, tập dữ liệu thích hợp để phân tích EFA. Biến (TR4) tải lên 2 nhân tố với hệ số tải lần lượt là 0,611 và 0,535 , biến (TR11) tải lên 2 nhân tố với hệ số tải lần lượt là 0,727 và 0,508 , biến (TR16) tải lên 2 nhân tố với hệ số tải lần lượt là 0,527 và 0,608 , nên loại 3 biến này khỏi thang đo, tập dữ liệu còn lại 25 biến. Phân tích $\mathrm{EFA}$ lần thứ ba cho hệ số $\mathrm{KMO}=0,812$ nằm trong giới hạn $[0,5 ; 1](\mathrm{p}=$ $0,000)$, rút trích được 4 nhân tố gồm 25 biến với tổng phương sai trích bằng $56,55 \%>50 \%$, giá trị Eigenvalue $>1$ và khác biệt hệ số tải nhân tố của một biến với các nhân tố khác đều $>0,3$.

\subsubsection{Phân tích nhân tố khẳng định}

Tiếp tục phân tích nhân tố khẳng định (CFA) để kiểm định thang đo. Lần chạy thứ nhất cho thấy biến (PE2) có trọng số $0,402<0,5$, biến (TR13) có trọng số $0,348<0,5$ nên để thang đo đạt giá trị hội tụ, loại 2 biến này khỏi thang đo. Lần chạy thứ hai cho thấy biến (PE4) có trọng số $0,348<0,5$, biến (PU3) có trọng số $0,382<0,5$ và biến (TR6) có trọng số $0,368<0,5$ nên loại 3 biến này khỏi thang đo. Tiếp tục chạy $\mathrm{CFA}$ lần thứ ba, dựa trên giá trị $\mathrm{MI}$ để lần lượt loại 3 biến (PE5), (TR8), (PU2). Thang đo sau khi cải thiện đạt được độ tương thích với dữ liệu, với các chỉ số Chi-square $(\mathrm{X} 2) / \mathrm{dF}=1.672(<2)(\mathrm{p}=0.000) ; \mathrm{CFI}=0.935(>0,9) ; \mathrm{TLI}=0.921(>0,9)$; RMSEA = 0.050 (<0,05) (Byrne, 2010; Hair, Black, Babin, \& Anderson, 2014). Hệ số tải chuẩn hóa của các biến có giá trị từ 0,690 đến 0,843 (đều >0,5). Độ tin cậy tổng hợp $(\mathrm{CR})$ có giá trị từ 0,679 đến 0,821 (đều $>0,5$ ) nên các thành phần thang đo đạt độ tin cậy. Phương sai trích trung bình $(\mathrm{AVE})$ có giá trị từ 0,513 đến 0,654 (đều $>0,5)$ nên các thành phần thang đo đạt giá trị hội tụ. Giá trị phân biệt của các khái niệm cũng đạt khi AVE đều lớn hơn bình phương tương quan giữa các khái niệm (Fornell \& Larcker, 1981). Như vậy, thang đo của nhân tố "sự sẵn sàng công nghệ" gồm 9 biến, thang đo của nhân tố "cảm nhận về tính dễ sử dụng" gồm 3 biến, thang đo của nhân tố "cảm nhận về tính hữu ích" gồm 4 biến, thang đo của nhân tố "ý định tiếp tục sử dụng công nghệ “gồm 2 biến; đều đạt độ tin cậy và độ giá trị. 


\section{Bảng 1}

Kết quả phân tích EFA và CFA

\begin{tabular}{|c|c|c|c|}
\hline Biến & Mô tả biến & $\begin{array}{c}\text { Hệ số tải } \\
\text { EFA }\end{array}$ & $\begin{array}{c}\text { Hệ số } \\
\text { tải } \\
\text { CFA }\end{array}$ \\
\hline \multicolumn{4}{|c|}{ Sự sẵn sàng công nghệ (TR): AVE $=0,541(>0,50) ; C R=0,722(>0,50)$} \\
\hline TR1 & Công nghệ giúp nâng cao chất lýợng cuộc sống & Loại bỏ & \\
\hline TR2 & Công nghệ mang dến sự tự do và quyền di động & 0,765 & 0,725 \\
\hline TR3 & Công nghệ giúp kiểm soát cuộc sống hàng ngày & 0,745 & 0,724 \\
\hline TR4 & Công nghệ giúp tãng nãng suất trong cuộc sống & Loại bỏ & \\
\hline TR5 & Những ngýời khác tìm tới tôi để dýợc tý vấn về công nghệ & 0,833 & 0,961 \\
\hline TR6 & Tôi là ngýời đầu tiên trong số bạn bè sở hữu công nghệ mới & 0,719 & 0,631 \\
\hline TR7 & Tôi có thế tự tìm ra các sản phẩm và dịch vụ công nghệ cao & Loại bỏ & \\
\hline TR8 & $\begin{array}{l}\text { Tôi theo kịp sự phát triển công nghệ trong các lĩnh vực quan } \\
\text { tâm }\end{array}$ & 0,617 & Loại bỏ \\
\hline TR9 & Tôi không cảm thấy bị ngýời hỗ trợ kỹ thuật lợi dụng & 0,828 & 0,833 \\
\hline TR10 & Sự hỗ trợ kỹ thuật là hữu ích & 0,768 & 0,754 \\
\hline TR11 & Ngýời bình thýờng có thể sử $\mathrm{d}$ & Loại bỏ & \\
\hline TR12 & Ngôn ngữ hỗ trợ kỹ thuật là đõn giản & 0,768 & 0,754 \\
\hline TR13 & Con ngýời không quá phụ thuộc vào công nghệ & 0,699 & Loại bỏ \\
\hline TR14 & $\begin{array}{l}\text { Con ngýời không bị ảnh hýởng xấu nếu sử dụng nhiều công } \\
\text { nghệ }\end{array}$ & 0,721 & 0,716 \\
\hline TR15 & Công nghệ không làm giảm týõng tác giữa ngýời và ngýời & 0,793 & 0,787 \\
\hline TR16 & $\begin{array}{l}\text { Tôi không cảm thấy mất tự tin khi làm việc với ngýời khác từ } \\
\text { xa }\end{array}$ & Loại bỏ & \\
\hline \multicolumn{4}{|c|}{ Cảm nhận về tính dễ sử dụng (PE): $\mathrm{AVE}=0,654(>0,50) ; \mathrm{CR}=0,843(>0,50)$} \\
\hline PE1 & Dễ học cách sử dụng mobile video & 0,841 & 0,862 \\
\hline PE2 & Dễ dùng mobile video cho những việc cần dùng & 0,741 & Loại bỏ \\
\hline PE3 & Mobile video rõ ràng và dễ hiểu & 0,805 & 0,765 \\
\hline PE4 & Mob & 0, & Loại bỏ \\
\hline PE5 & Dễ đạ & 0,656 & Loại bỏ \\
\hline PE6 & Mobile video dễ sử dụng & 0,775 & 0,701 \\
\hline \multicolumn{4}{|c|}{ Cảm nhận về tính hữu ích $(\mathrm{PU})$ : AVE = 0,513 (> 0,50); $\mathrm{CR}=0,725(>0,50)$} \\
\hline PU1 & Mobile video giúp hoàn thành nhiệm vụ học tập nhanh hơn & 0,783 & 0,822 \\
\hline PU2 & Mobile vi & 0,676 & Loại bỏ \\
\hline PU3 & Mobile video giúp tăng năng suất trong học tập & 0,559 & Loại bỏ \\
\hline PU4 & Mobile video giúp nâng cao hiệu quả học tập & 0,716 & 0,717 \\
\hline PU5 & Mobile video giúp việc học tập dễ dàng hơn & 0,757 & 0,744 \\
\hline PU6 & Mobile video hữu dụng cho việc học tập & 0,689 & 0,640 \\
\hline \multicolumn{4}{|c|}{ Ý định sử dụng công nghệ (IU): AVE = 0,630 (>0,50); $\mathrm{CR}=0,734(>0,50)$} \\
\hline IU1 & Tôi sẽ sử dụng mobile video trong những lần học sau & 0,798 & 0,833 \\
\hline IU2 & Tôi sẽ sử dụng mobile video trong vài tháng tới & 0,624 & 0,628 \\
\hline
\end{tabular}

Nguồn: Kết quả phân tích dữ liệu của nhóm nghiên cứu 


\subsubsection{Phân tích mô hình cấu trúc tuyến tính}

Phân tích mô hình cấu trúc tuyến tính ( $\mathrm{SEM})$ để kiểm tra cấu trúc mô hình, bằng ước lượng ML, kết quả cho thấy mô hình đạt độ phù hợp chung với Chi-square $(\mathrm{X} 2) / \mathrm{dF}=1,671(<2)$ (với $\mathrm{p}=0,000)$; $\mathrm{CFI}=0,934(>0,9)$; TLI $=0,934(>0,9)$; RMSEA $=0,050(<0,05)$ (Byrne, 2010; Hair et al., 2014). Có 5 giả thuyết được ủng hộ và 1 giả thuyết bị bác bỏ (xem Bảng 2). Như vậy, TR có tác động tích cực lên cả $\mathrm{PE}$ và $\mathrm{PU}$; IU bị ảnh hưởng tích cực bởi $\mathrm{PE}$ và $\mathrm{PU}$; $\mathrm{PE}$ có tác động tích cực lên PU.

\section{Bảng 2}

Kết quả phân tích SEM

\begin{tabular}{|c|c|c|c|c|}
\hline Giả thuyết & Quan hệ & Ước lượng & Mức ý nghĩa & Kết quả \\
\hline H1 & PE -> IU & 0,451 & 0,001 & Ủng hộ \\
\hline H2 & PU -> IU & 0,671 & $* * *$ & Úng hộ \\
\hline H3 & PE -> PU & 0,790 & $* * *$ & Úng hộ \\
\hline H4 & TR -> IU & 0,346 & 0,209 & Bác bỏ \\
\hline H5 & TR -> PE & 0,684 & 0,001 & Úng hộ \\
\hline H6 & TR -> PU & 0,542 & 0,005 & Ủng hộ \\
\hline
\end{tabular}

Nguồn: Kết quả phân tích dữ liệu của nhóm nghiên cứu

\section{Kết luận và kiến nghị}

\subsection{Nhũng phát hiện chính}

Nghiên cứu này áp dụng mô hình TRAM của Lin và cộng sự (2007) để kiểm tra ảnh hưởng của một số yếu tố liên quan đến niềm tin và thái độ lên ý định sử dụng mobile video để học tập trực tuyến. Đối tượng khảo sát là sinh viên Đại học Bách khoa TP.HCM. Kết quả cho thấy, thứ nhất, những sinh viên có ý định sử dụng mobile video để việc học tập trực tuyến sẽ bị ảnh hưởng tích cực bởi cảm nhận về tính dễ sử dụng và cảm nhận về tính hữu ích của mobile video, trong đó, cảm nhận về tính dễ sử dụng có ảnh hưởng mạnh hơn. Thứ hai, có một tác động tích cực đáng kể của cảm nhận về tính dễ sử dụng của mobile video lên cảm nhận về tính hữu ích của mobile video. Những điều này có thể được lý giải là một sản phẩm công nghệ có thể không thu hút sự chú ý của người dùng nếu nó không dễ sử dụng. Nếu người dùng có các kỹ năng cần thiết để sử dụng một sản phẩm công nghệ mới, họ sẽ tự nâng cao nhận thức về tính hữu ích của sản phẩm đó và có nhiều khả năng sẽ lựa chọn sử dụng sản phẩm đó. Những phát triển gần đây về cơ sở hạ tầng viễn thông và sự sẵn có của nhiều thương hiệu điện thoại thông minh giá rẻ trên thị trường Việt Nam đã giúp người học có cơ hội sở hữu và sử dụng điện thoại thông minh cho nhiều mục đích, trong đó có học tập trực tuyến. Sản phẩm điện thoại thông minh nào càng dễ sử dụng, các chương trình đào tạo trực tuyến bằng mobile video nào càng dễ tiếp cận và đơn giản khi tương tác thì sẽ càng giúp người học đánh giá cao về tính hữu ích. Và khi cảm nhận về tính dễ sử dụng lẫn tính hữu ích càng gia tăng thì người học càng gia tăng ý định sử dụng.

Thứ ba, có một tác động mạnh mẽ của sự sẵn sàng công nghệ của người học lên cảm nhận của họ về tính dễ sử dụng và cảm nhận về tính hữu ích của mobile video. Điều này ngụ ý rằng nếu người học có những niềm tin tích cực về công nghệ thì họ sẽ có khuynh hướng đánh 
giá điện thoại thông minh và các chương trình đào tạo trực tuyến bằng mobile video không khó để sử dụng, và sẽ nhìn nhận tính hữu ích của các chương trình đào tạo trực tuyến bằng mobile video. Từ đó, họ càng gia tăng khả năng chấp nhận sử dụng mobile video để học tập trực tuyến trong tương lai.

\subsection{Kiến nghị hướng nghiên cứu tiếp theo}

Thứ nhất, nghiên cứu này bị giới hạn bởi kỹ thuật lấy mẫu phi xác suất. Thứ hai, chỉ có sự sẵn sàng công nghệ được đưa vào mô hình nghiên cứu như một nhân tố ảnh hưởng đến cảm nhận về tính dễ sử dụng và cảm nhận về tính hữu ích, trong khi thực tế, có thể có một số yếu tố khác có thể ảnh hưởng, chẳng hạn như ảnh hưởng xã hội, các điều kiện hỗ trợ... Do đó, hướng phát triển tiếp theo của nghiên cứu này là sử dụng các phương pháp lấy mẫu tin cậy hơn, và tiếp cận các khái niệm liên quan một cách đa chiều để có cái nhìn sâu và rộng hơn trong việc xem xét các tiền tố về niềm tin và thái độ đối với ý định sử dụng mobile video trong học tập trực tuyến. Ngoài ra, có thể bổ sung thêm các nhân tố mới vào mô hình, bổ sung các biến đo lường mới vào thang đo, thực hiện thêm bước nghiên cứu định tính và mở rộng nghiên cứu trong nhiều bối cảnh khác nhau, từ đó thu được mô hình và thang đo đủ độ tin cậy và độ giá trị, giúp dự đoán và kiểm soát ý định sử dụng mobile video trong học tập trực tuyến tại Việt Nam thông qua các tiền tố quan trọng đã được kiểm định.

\section{LÒ̀I CẢM ƠN}

Nghiên cứu này được tài trợ bởi Trường Đại học Bách Khoa - ĐHQG-HCM trong khuôn khổ đề tài mã số To-QLCN-2017-16.

\section{Tài liệu tham khảo}

Adiyarta, K., Napitupulu, D., Nurdianto, H., Rahim, R., \& Ahmar, A. (2018). User acceptance of e-government services based on TRAM model. Paper presented at the 7th AIC-ICMR on Sciences and Engineering 2017, Banda Aceh, Indonesia.

Almaiah, M. A., \& Alismaiel, O. A. (2018). Examination of factors influencing the use of mobile learning system: An empirical study. Education and Information Technologies, 23(6), 1-25.

Ambient Insight. (2014). The 2013-2018 Asia self-paced eLearning market. Retrieved October 5, 2017, from Ambient Insight website: http://www.ambientinsight.com/Resources/Documents/AmbientInsight-2013-2018Asia-Self-paced-eLearning-Market-Executive-Overview.pdf

AUM Việt Nam. (2017). Thị trương giáo dục trục tuyến - Việt Nam đứng TOP tại châu Á [Online education market - Vietnam ranks TOP in Asia]. Retrieved October 20, 2017, from http://aum.edu.vn/tin-tuc/thi-truong-giao-duc-truc-tuyen-viet-nam-dung-top-taichau-a.html

Báo Dân Trí. (2017). Đâu sẽ trở thành xu hướng học trục tuyến nổi bật 2017? [What will become the outstanding online learning trends in 2017?]. Retrieved October 25, 2017, 
from Dân Trí website: https://dantri.com.vn/tin-tuyen-sinh/dau-se-tro-thanh-xu-huonghoc-truc-tuyen-noi-bat-2017-20171004182807314.htm

Báo điện tử Tri Thức Trẻ. (2017). Việt Nam thuộc top 10 thị trương giáo dục trục tuyến năng động tại châu A [Vietnam is in the top 10 dynamic online education markets in Asia]. Retrieved October 27, 2017, from Báo điện tử Tri Thức Trẻ website: http://kenh14.vn/viet-nam-thuoc-top-10-thi-truong-giao-duc-truc-tuyen-nang-dong-taichau-a-20170601064156291.chn

Báo Nhân Dân. (2017). Đào tạo trục tuyến trong thời kỳ cách mạng công nghiệp 4.0 [Online training during the industrial revolution 4.0]. Retrieved October 28, 2017, from Báo Nhân Dân website: http://www.nhandan.com.vn/giaoduc/dien-dan/item/31943302-daotao-truc-tuyen-trong-thoi-ky-cach-mang-cong-nghiep-4-0.html

Baturay, M. H., Gökçearslan, Ş., \& Ke, F. (2017). The relationship among pre-service teachers' computer competence, attitude towards computer-assisted education, and intention of technology acceptance. International Journal of Technology Enhanced Learning, 9(1), $1-13$.

Byrne, B. M. (2010). Structural equation modeling with AMOS: Basic concepts, applications, and programming (2nd ed.). New York, NY: Routledge Taylor and Francis Group, LLC.

Carter, V. (1996). Do media influence learning? Revisiting the debate in the context of distance education. Open Learning: The Journal of Open, Distance and e-Learning, 11(1), 31-40.

Cheon, J., Lee, S., Crooks, S. M., \& Song, J. (2012). An investigation of mobile learning readiness in higher education based on the theory of planned behavior. Computers \& Education, 59(3), 1054-1064.

Christensen, R., \& Knezek, G. (2017). Readiness for integrating mobile learning in the classroom: Challenges, preferences and possibilities. Computers in Human Behavior, 76, 112-121.

Chung, S., Lee, K. Y., \& Choi, J. (2015). Exploring digital creativity in the workspace: The role of enterprise mobile applications on perceived job performance and creativity. Computers in Human Behavior, 42, 93-109.

Cortina, J. M. (1993). What is coefficient alpha? An examination of theory and applications. Journal of Applied Psychology, 78(1), 98-104.

Crosbie, T., Broderick, J., Short, M., Charlesworth, R., \& Dawood, M. (2018). Demand response technology readiness levels for energy management in blocks of buildings. Buildings, 8(2), 13.

Das, J. R., Dash, M., Sahoo, M. A., \& Mohanty, A. K. (2017). An empirical study on customers' Internet banking behavior. International Journals of Multidisciplinary Research Academy, 7(7), 218-226.

Davis, F. D. (1989). Perceived usefulness, perceived ease of use, and user acceptance of information technology. MIS Quarterly, 13(3), 319-340.

Fornell, C., \& Larcker, D. F. (1981). Evaluating structural equation models with unobservable variables and measurement error. Journal of Marketing Research, 18(1), 39-50. 
Gruzd, A., Staves, K., \& Wilk, A. (2012). Connected scholars: Examining the role of social media in research practices of faculty using the UTAUT model. Computers in Human Behavior, 28(6), 2340-2350.

Guo, Y., \& Barnes, S. J. (2012). Explaining purchasing behavior within world of Warcraft. Journal of Computer Information Systems, 52(3), 18-30.

Haag, J. (2011). From eLearning to mLearning: The effectiveness of mobile course delivery. Paper presented at the Interservice/Industry Training, Simulation \& Education Conference (I/ITSEC).

Hair, J. F., Tatham, R. L., \& Black, W. C. (1998). Multivariate data analysis. Upper Saddle River, NJ: Prentice-Hall, Inc.

Hair, J. F., Black, W. C., Babin, B. J., \& Anderson, R. E. (2014). Multivariate data analysis. (7th ed., Pearson New International Edition). Harlow, UK: Pearson Education Limited.

Hastings, N. B., \& Tracey, M. W. (2005). Does media affect learning: Where are we now? TechTrends, 49(2), 28-30.

Hong, W., Thong, J. Y., Chasalow, L. C., \& Dhillon, G. (2011). User acceptance of agile information systems: A model and empirical test. Journal of Management Information Systems, 28(1), 235-272.

Howard, S. K., Chan, A., \& Caputi, P. (2015). More than beliefs: Subject areas and teachers' integration of laptops in secondary teaching. British Journal of Educational Technology, 46(2), 360-369.

Huang, F., Sánchez-Prieto, J., Teo, T., Olmos-Migueláñez, S., \& García-Peñalvo, F. (2018). ICT acceptance among university teachers: A cross-cultural comparison between China and Spain. Paper presented at the ECER'18 Conference, Bolzano, Italy.

Hussin, S., Manap, M. R., Amir, Z., \& Krish, P. (2012). Mobile learning readiness among Malaysian students at higher learning institutes. Asian Journal of Social Science, 8(12), 276-283.

Iqbal, S., \& Bhatti, Z. A. (2015). An investigation of university student readiness towards mlearning using technology acceptance model. The International Review of Research in Open and Distributed Learning, 16(4), 83-103.

Irby, D. R. (2017). Middle school student and teacher perceptions about the effectiveness of the technology integration in the classroom (Doctoral dissertation, University of MissouriSt. Louis, USA). Retrieved May 5, 2017, from https://irl.umsl.edu/dissertation/646/

Jin, C. (2013). The perspective of a revised TRAM on social capital building: The case of Facebook usage. Information \& Management, 50(4), 162-168.

Khaddage, F., \& Knezek, G. (2013). iLearn via mobile technology: A comparison of mobile learning attitudes among university students in two nations. Paper presented at the Advanced Learning Technologies (ICALT), 2013 IEEE 13th International Conference on. 
Khaddage, F., Christensen, R. R., Lai, W., Knezek, G., Norris, C., \& Soloway, E. (2015). A model driven framework to address challenges in a mobile learning environment. Education and Information Technologies, 20(4), 625-640.

Larasati, N., \& Santosa, P. I. (2017). Technology readiness and technology acceptance model in new technology implementation process in low technology SMEs. International Journal of Innovation, Management and Technology, 8(2), 113-117.

Lian, J.-W., Yen, D. C., \& Wang, Y.-T. (2014). An exploratory study to understand the critical factors affecting the decision to adopt cloud computing in Taiwan hospital. International Journal of Information Management, 34(1), 28-36.

Liew, C. S., Ang, J. M., Goh, Y. T., Koh, W. K., Tan, S. Y., \& Teh, R. Y. (2017). Factors influencing consumer acceptance of internet of things technology. In Handbook of Research on Leveraging Consumer Psychology for Effective Customer Engagement (pp. 186-201). Hershey, PA: IGI Global.

Lin, C. H., Shih, H. Y., \& Sher, P. J. (2007). Integrating technology readiness into technology acceptance: The TRAM model. Psychology \& Marketing, 24(7), 641-657.

Lundberg, E. (2017). How to compete effectively with self-service technologies: The impact of technology readiness and the technology acceptance model on self-scanning (Master's thesis, Uppsala University, Sweden). Retrieved May 10, 2017, from http://www.divaportal.org/smash/get/diva2:1111218/FULLTEXT01.pdf

Mahat, J., Ayub, A. F. M., \& Luan, S. (2012). An assessment of students' mobile self-efficacy, readiness and personal innovativeness towards mobile learning in higher education in Malaysia. Procedia-Social and Behavioral Sciences, 64, 284-290.

Maruping, L., Magni, M., Caporarello, L., \& Basaglia, S. (2008). What's the weather like? The effect of team climate and individual attributes on individual intention to explore a new technology. AMCIS 2008 Proceedings, 383.

Maruping, L. M., \& Magni, M. (2012). What's the weather like? The effect of team learning climate, empowerment climate, and gender on individuals' technology exploration and use. Journal of Management Information Systems, 29(1), 79-114.

Nunnally, J. (1978). Psychometric methods. New York, NY: McGraw-Hill.

Parasuraman, A. (2000). Technology Readiness Index (TRI) a multiple-item scale to measure readiness to embrace new technologies. Journal of Service Research, 2(4), 307-320.

Parasuraman, A., \& Colby, C. L. (2015). An updated and streamlined technology readiness index: TRI 2.0. Journal of Service Research, 18(1), 59-74.

Park, E., Cho, Y., Han, J., \& Kwon, S. J. (2017). Comprehensive approaches to user acceptance of Internet of things in a smart home environment. IEEE Internet of Things Journal, 4(6), 2342-2350.

Pynoo, B., Devolder, P., Tondeur, J., van Braak, J., Duyck, W., \& Duyck, P. (2011). Predicting secondary school teachers' acceptance and use of a digital learning environment: A cross- 
sectional study. Computers in Human Behavior, 27(1), 568-575. doi:10.1016/j.chb.2010.10.005

Rivera, J., \& van der Meulen, R. (2014). Gartner says annual smartphone sales surpassed sales of feature phones for the first time in 2013. England, UK: Egham.

Sánchez-Prieto, J., Olmos-Migueláñez, S., \& García-Peñalvo, F. (2017a). Technology acceptance among teachers: An SLR on TAM and teachers. Retrieved May 12, 2017, from https://konferens.ht.lu.se/fileadmin/_migrated/content_uploads/SanchezPrieto_etal.pdf

Sánchez-Prieto, J., Olmos-Migueláñez, S., \& García-Peñalvo, F. (2017b). MLearning and preservice teachers: An assessment of the behavioral intention using an expanded TAM model. Computers in Human Behavior, 72, 644-654.

Scherer, R., Siddiq, F., \& Tondeur, J. (2019). The technology acceptance model (TAM): A meta-analytic structural equation modeling approach to explaining teachers' adoption of digital technology in education. Computers \& Education, 128, 13-35.

Van Der Molen, J. H. W., \& Van Der Voort, T. H. (2000). The impact of television, print, and audio on children's recall of the news. A study of three alternative explanations for the dual- coding hypothesis. Human Communication Research, 26(1), 3-26.

Venkatesh, V., Thong, J. Y. L., Chan, F. K. Y., Hu, P. J.-H., \& Brown, S. A. (2011). Extending the two-stage information systems continuance model: Incorporating UTAUT predictors and the role of context. Information Systems Journal, 21(6), 527-555. doi:10.1111/j.13652575.2011.00373.x

Wingo, N. P., Ivankova, N. V., \& Moss, J. A. (2017). Faculty perceptions about teaching online: Exploring the literature using the technology acceptance model as an organizing framework. Online Learning, 21(1), 15-35. 